\title{
POLYNOMIAL RINGS WITH A PIVOTAL MONOMIAL ${ }^{1}$
}

\section{S. K. JAIN ${ }^{2}$}

1. Amitsur in his paper on Finite Dimensional Central Division Algebras [1] has proved that in a division ring $D$ with center $C$, $(D: C) \leqq n^{2}<\infty$ if and only if every primitive homomorphic image of a polynomial ring $D[x]$ is a complete matrix ring $A_{h}, h \leqq n$, over a division ring $A$. Equivalently speaking, a division ring is finite dimensional over its center if and only if the polynomial ring over it has a $J$-pivotal monomial (written as JPM). The object of this note is to show that if $R$ is a ring with a nilpotent (Jacobson) radical then the polynomial ring $R[x]$ has a JPM if and only if $R[x]$ has a polynomial identity. Amitsur's result then follows as a special case of our result. Our proof of Theorem 1, in obtaining sufficiency, is on the same lines as that of Amitsur.

2. We begin with

Theorem 1. Let $R$ be a primitive algebra over its centroid $C$. Then $(R: C) \leqq n^{2}<\infty$ if and only if every primitive homomorphic image of $R[x]$ is a complete matrix ring $A_{h}, h \leqq n$, over a division ring $A$.

Proof of the theorem: Necessity. Let $(R: C) \leqq n^{2}<\infty$. Then it is well known that $R$ satisfies a minimal polynomial identity $S_{d}(x)$ $=\sum \pm x_{i_{1}} x_{i_{2}} \cdots x_{i_{d}}$, of degree $d \leqq 2 n$. This identity also holds in $R[x]$. Since a primitive ring with a polynomial identity of degree $d$ is a central simple algebra with a dimensionality $\leqq[d / 2]^{2}$, it follows that each primitive homomorphic image of $R[x]$ is a central simple algebra of dimension $\leqq[d / 2]^{2}$; and therefore it is isomorphic to $A_{r}$ for some division algebra $A$ and for $r \leqq d / 2 \leqq n$. This proves necessity.

Before we obtain sufficiency we recall for convenience the definition of a $J$-pivotal monomial in a ring. Let $\lambda_{1}, \cdots, \lambda_{t}$ be a set of noncommutative indeterminates and let $\pi(\lambda)=\lambda_{i_{1}} \cdots \lambda_{i_{d}}$ be a monomial of degree $d$ in the $\lambda_{i}$. Let $P_{\pi}$ denote the set of all monomials $\sigma(\lambda)=\lambda_{j_{1}} \cdots \lambda_{j_{q}}$ such that either $q>d$ or $q \leqq d$ with $j_{h} \neq i_{h}$ for some $h \leqq q$. We call a monomial $\pi(\lambda)$ a right $J$-pivotal monomial for a ring $R$ if for every substitution $\lambda_{i}=x_{i} \in R, \pi(x) r$ is right-quasi-regular

Received by the editors February 12, 1965.

1 Research partially supported by NSF GP-1447.

${ }^{2}$ The author wishes to express his thanks to Professor S. A. Amitsur for reading the original manuscript and for his valuable comments. 
$\bmod \sum_{\sigma \in P \pi} \sigma(x) R$, for all $r \in R$. A ring with a right $J$-pivotal monomial is called a right JPM-ring. Henceforth a JPM-ring shall mean a right JPM-ring. It is proved in [2] that a ring $R$ has a $J$-pivotal monomial of degree $d$ if and only if every (right) primitive homomorphic image of $R$ is a full matrix ring $D_{h}$ over a division ring $D$ with $h \leqq d$. A simple but an important consequence of the definition of a JPM-ring may be recorded in

Sublemma. A homomorphic image of a JPM-ring is also a JPMring. In particular, if $R[x]$ has a JPM then its homomorphic image $R$ is also a JPM-ring.

Sufficiency. Let $R$ be a primitive ring such that every primitive homomorphic image of $R[x]$ is a complete matrix ring $A_{h}, h \leqq n$, over a division ring $A$, viz., $R[x]$ has a JPM of degree $n$. So that by the sublemma $R$ has JPM of degree $n$ and consequently, it is full matrix ring $A_{h}, h \leqq n$ over a division ring $A$. Therefore we have

$$
R[x]=A_{h}[x] \cong(A[x])_{h} .
$$

We can assume that

$$
R[x]=(A[x])_{h}=S_{h}, \quad S=A[x] .
$$

Consider the maximal right ideal

$$
I=(x-a) A[x], \quad a \in A .
$$

We note that each primitive ideal of $A[x]$ will be maximal ideal of $A[x]$. Therefore if $P=p(x) A[x]$ ( $A[x]$ is a principal ideal ring) be a primitive ideal contained in $I$, then $P$ is a maximal ideal in $A[x]=S$. Since $S$ has unity, $S / P$ is a simple primitive ring. Then the isomorphism

$$
S_{h} / P_{h} \cong(S / P)_{h}
$$

gives that $S_{h} / P_{h}$ is a primitive ring. Accordingly, $S_{h} / P_{h} \cong D_{r}$ with $r \leqq n$. Further if $I_{u}=\left(x-u a u^{-1}\right) A[x], 0 \neq u \in A$, then it can be verified that

$$
P_{h}=\bigcap\left(I_{u}\right)_{h} .
$$

Since $S_{h} / P_{h} \cong D_{r}$, we can find $r$ elements $u_{1}, \cdots, u_{r}$ such that

$$
\begin{aligned}
A_{h}[x] & \supset\left(I_{u_{1}}\right)_{h} \supset\left(I_{u_{1}}\right)_{h} \cap\left(I_{u_{2}}\right)_{h} \supset \cdots \\
& \supset\left(I_{u_{1}}\right)_{h} \cap\left(I_{u_{2}}\right)_{h} \cap \cdots \cap\left(I_{u_{r}}\right)_{h}=P_{h} .
\end{aligned}
$$

Observing that $\left(I_{u}\right)_{h}=\left(x-u a u^{-1}\right) A_{h}[x]$, we can claim that $p(x)$ is a left common divisor of polynomials $x-u_{i} a u_{i}^{-1}$ and therefore degree of 
$p(x) \leqq r$. It follows therefore that for each $a$ in $A$ there exists a polynomial $p(x)$ of degree $\leqq n$ with coefficients in center such that $x-a$ is a right divisor of $p(x)$. Hence $p(a)=0$. This implies $A$ is an algebraic algebra of bounded degree. By Kaplansky [5] $A$ satisfies a polynomial identity and is finite dimensional over its center. Hence $R=A_{h}$ is finite dimensional over its center (=centroid, since $R$ has a unity). This completes the proof.

Next we prove

Theorem 2. Let $R$ be a ring having its (Jacobson) radical nilpotent. Then $R[x]$ has JPM if and only if $R[x]$ has $P I$.

Proof : NeCEssity. Let $J$ be radical of $R$ and $J^{m}=0$. Let $R[x]$ have JPM of degree $n$. Let $\bar{P}$ be a primitive homomorphic image of $\bar{R}=R / J$. Then this, along with natural homomorphism induces the diagram

$$
R[x] \rightarrow \bar{R}[x] \rightarrow \bar{P}[x] .
$$

By the sublemma $\bar{P}[x]$ has JPM and therefore Theorem 1 gives that $\bar{P}$ satisfies a standard identity of degree $\leqq 2 n$. Consequently, $\bar{R}$ which is a subdirect sum of its primitive images satisfies a standard identity $S_{d}(x)=0$ of degree $d \leqq 2 n$. This implies $R$ satisfies $\left[S_{2 n}(x)\right]^{m}=0$. The sufficiency is easy and therefore omitted.

REMARK 1. The theorem is still true for a ring $R$ having its radical satisfying some polynomial identity. For if $J$ satisfies an identity $p\left(x_{1}, \cdots, x_{k}\right)=0$, then $R$ will satisfy $p\left[S_{2 n}\left(x_{1}^{\prime}, \cdots, x_{2 n}^{\prime}\right), \cdots\right.$, $\left.\mathrm{S}_{2 n}\left(x_{1}^{\mathbf{k}}, \cdots, x_{2 n}^{\mathbf{k}}\right)\right]=0$.

REMARK 2. The theorem is also true for a ring $R$ with a strongly pivotal monomial and nil radical. For, in this case, radical will be nilpotent.

Belluce and Jain [3] have shown that a primitive ring satisfies a polynomial identity if and only if (1) it has at most a finite number of orthogonal idempotents (written as FI-ring), and (2) it has a nonzero one-sided ideal satisfying some polynomial identity. This result along with Theorem 2 gives the following,

THEOREM 3. Let $R$ be a primitive algebra over its centroid $C$. Then $(R: C) \leqq n^{2}<\infty$ if and only if $R$ is an FI-ring having a nonzero onesided ideal $I$ such that every primitive homomorphic image of $I[x]$ is a complete matrix ring $A_{h}, h \leqq n$, over a division ring $A$.

\section{BIBLIOGRAPHY}

1. S. A. Amitsur, Finite dimensional central division algebras, Proc. Amer. Math. Soc. 11 (1960), 28-31. 

642.

2. ——, Rings with a pivotal monomial, Proc. Amer. Math. Soc. 9 (1958), 635-

3. L. P. Belluce and S. K. Jain, Prime rings having a one-sided ideal satisfying a polynomial identity, Abstract 614-89, Notices Amer. Math. Soc. 11 (1964), p. 554.

4. N. Jacobson, Structure of rings, Amer. Math. Soc. Colloq. Publ. Vol. 37, Amer. Math. Soc., Providence, R. I., 1956.

5. I. Kaplansky, Rings with a polynomial identity, Bull. Amer. Math. Soc. 54 (1948), 575-580.

University of California, Riverside

\section{CORRECTION TO “ON MATRICES WHOSE REAL LINEAR COMBINATIONS ARE NONSINGULAR"}

\section{J. F. ADAMS, PETER D. LAX AND RALPH S. PHILLIPS}

We are grateful to Professor B. Eckmann for pointing out an error in the proof of Lemma 4(b) of our paper [1]. This error invalidates Lemma 4(b) and that part of Theorem 1 which states the values of $Q(n), Q_{H}(n)$. The error occurs immediately after the words "arguing as is usual for the complex case, we find"; it consists in manipulating as if the ground field $\Lambda$ were commutative.

The proof of Lemma 4(b) can be repaired, as will be shown below, but it leads to a different conclusion from that given. Our paper should therefore be corrected as follows.

(i) In Theorem 1, the values of $Q(n)$ and $Q_{H}(n)$ should read

$$
\text { “ } Q(n)=\rho\left(\frac{1}{2} n\right)+4, \quad Q_{H}(n)=\rho\left(\frac{1}{4} n\right)+5 . "
$$

The two sentence paragraph following Theorem 1 should be deleted. It remains interesting to ask what topological phenomena (if any) can be related to our algebraic results.

(ii) In Lemma 4, part (b) should read

$$
\text { "Q } Q_{H}(n)+3 \leqq R(4 n) . "
$$

The proof is as follows.

Let $W$ be a $k$-dimensional space of $n \times n$ Hermitian matrices with entries from $Q$ which has the property $P$. The space $Q^{n}$ is a real vector space of dimension $4 n$. For each $A \in W$ and each pure imaginary $\mu \in Q$ we consider the following real-linear transformation from $Q^{n}$ to itself:

Received by the editors December 20, 1965. 